\title{
Multiplicity of Homoclinic Solutions for a Class of Nonperiodic Fourth-Order Differential Equations with General Perturbation
}

\author{
Liu Yang \\ Department of Mathematics and Computing Sciences, Hengyang Normal University, Hengyang, Hunan 421008, China \\ Correspondence should be addressed to Liu Yang; yangliu19731974@yahoo.com.cn \\ Received 14 April 2014; Accepted 17 June 2014; Published 7 July 2014 \\ Academic Editor: Jianqing Chen \\ Copyright (C) 2014 Liu Yang. This is an open access article distributed under the Creative Commons Attribution License, which \\ permits unrestricted use, distribution, and reproduction in any medium, provided the original work is properly cited. \\ In this paper, we investigate a class of nonperiodic fourth-order differential equations with general perturbation. By using the \\ mountain pass theorem and the Ekeland variational principle, we obtain that such equations possess two homoclinic solutions. \\ Recent results in the literature are generalized and significantly improved.
}

\section{Introduction}

In this paper, we consider the following class of fourth-order differential equations:

$$
\begin{aligned}
u^{(4)}+ & w u^{\prime \prime}+a(x) u \\
& =f(x, u)+\lambda g(x, u), \quad x \in \mathbb{R},
\end{aligned}
$$

where $w$ is a constant, $f \in C(\mathbb{R} \times \mathbb{R}, \mathbb{R}), g \in C(\mathbb{R} \times \mathbb{R}, \mathbb{R})$, and $a \in C(\mathbb{R}, \mathbb{R})$.

Recently, a lot of attention has been focused on the study of homoclinic and heteroclinic solutions for this problem; see [1-8]. This may be due to its concrete applications, such as physics and mechanics; see $[9,10]$. More precisely, Tersian and Chaparova [5] studied periodic case. They obtained nontrivial homoclinic solution by using mountain pass theorem. For nonperiodic case, Li [7] studied the existence of nontrivial homoclinic solution for this class of equations. Sun and $\mathrm{Wu}$ [8] studied multiple homoclinic solutions for the following nonperiodic fourth-order equations with a perturbation:

$$
\begin{array}{r}
u^{(4)}+w u^{\prime \prime}+a(x) u=f(x, u)+\lambda h(x)|u|^{p_{0}-2} u, \\
x \in \mathbb{R}, \quad 1 \leq p_{0}<2 .
\end{array}
$$

Before stating the results of [8] and our results, we introduce some notations. Throughout this paper, we denote by \|\|$_{r}$ the
$L^{r}$-norm, $2 \leq r \leq \infty . L^{\infty}(\mathbb{R})$ is the Banach space of essentially bounded functions equipped with the norm

$$
\|u\|_{\infty}=\operatorname{ess} \sup \{|u(x)|: x \in \mathbb{R}\} .
$$

If we take a subsequence of a sequence $\left\{u_{n}\right\}$, we will denote it again by $\left\{u_{n}\right\}$.

Theorem 1 (see [8]). For any real number $k \geq 1$, if the following conditions are satisfied,

$\left(A_{0}\right)$ there exists a positive constant $a_{1}$ such that $0<a_{1} \leq$ $a(x) \rightarrow+\infty$ as $|x| \rightarrow+\infty$ and $w \leq 2 \sqrt{a_{1}}$.

$\left(\mathrm{F}_{1}\right) f(x, s)$ is a continuous function on $\mathbb{R} \times \mathbb{R}$ such that $f(x, s) \equiv 0$ for all $s<0$ and $x \in \mathbb{R}$ and, moreover, there exists $b \in L^{\infty}\left(\mathbb{R}, \mathbb{R}^{+}\right)$with $|b|_{\infty}<c_{0} S_{2}^{2}$ such that

$$
\begin{gathered}
\lim _{s \rightarrow 0^{+}} \frac{f(x, s)}{s^{k}}=b(x) \quad \text { uniformly in } x \in \mathbb{R}, \\
\frac{f(x, s)}{s^{k}} \geq b(x) \quad \forall s>0, x \in \mathbb{R},
\end{gathered}
$$

where $c_{0}$ is defined by (12) in Section 2 and $S_{r}$ are the best constants for the embedding of $H^{2}(\mathbb{R})$ in $L^{r}(\mathbb{R})$ for $2 \leq r<$ $\infty$. that

$\left(\mathrm{F}_{2}\right)$ There exists $q \in L^{\infty}\left(\mathbb{R}, \mathbb{R}^{+}\right)$with $|q|_{\infty}>c_{0} S_{2}^{2}$ such

$$
\lim _{s \rightarrow \infty} \frac{f(x, s)}{s^{k}}=q(x) \quad \text { uniformly in } x \in \mathbb{R} .
$$


$\left(\mathrm{F}_{3}\right)$ There exist two constants $\theta, d_{0}$ satisfying $\theta>2$ and $0 \leq d_{0}<\left(c_{0} S_{2}^{2}(\theta-2) / 2 \theta\right)$ such that

$$
F(x, s)-\frac{1}{\theta} f(x, s) s \leq d_{0} s^{2} \quad \forall s>0, x \in \mathbb{R},
$$

where $F(x, s)=\int_{0}^{s} f(x, \tau) d \tau$, then one has the following results:

(i) if $k=1$ and $\mu<1$ with

$$
\begin{aligned}
\mu=\inf & \left\{\int_{\mathbb{R}}\left(u^{\prime \prime}(x)\right)^{2}-w u^{\prime}(x)^{2}\right. \\
& +a(x) u(x)^{2} d x \mid u \in H^{2}(\mathbb{R}), \\
& \left.\int_{\mathbb{R}} q(x) u(x)^{2} d x=1\right\},
\end{aligned}
$$

then there exists $\Lambda>0$ such that, for every $\lambda \in(0, \Lambda)$, problem (2) has at least two homoclinic solutions,

(ii) if $k>1$, then there exists $\Lambda>0$ such that, for every $\lambda \in(0, \Lambda)$, problem (2) has at least two homoclinic solutions.

The sublinear perturbation $h(x)|u|^{p-2} u, 1 \leq p<2$ is too strict; for example,

$$
g(x, u)= \begin{cases}h(x)|u|^{1 / 2}, & |u|<1, \\ h(x)|u|^{1 / 3}, & |u| \geq 1,\end{cases}
$$

does not satisfy this perturbation. However, one can see that our results in this paper can also work in this case. In addition, $\mu$ in Theorem 1 was defined in $\mathbb{R}$. Motivated by the above results, in this paper, we consider more general perturbation and the case of $\mu$ defined in local bounded open set $\Omega \subset \mathbb{R}$.

Now, we state our main result.

Theorem 2. For any real number $k \geq 1$, if $(A),\left(F_{1}\right)-\left(F_{3}\right)$ and the following conditions are satisfied,

$\left(G_{1}\right)$ there exist a constant $p \in(1,2)$ and a positive function $h \in L^{2 /(2-p)}\left(\mathbb{R}, \mathbb{R}^{+}\right)$such that

$$
|g(x, s)| \leq p h(x)|s|^{p-1},
$$

$\left(G_{2}\right)$ there exist a constant $\nu \in(1,2)$, a bounded open set $\Omega \subset$ $\mathbb{R}$, and a positive function $\xi \in C\left(\Omega, \mathbb{R}^{+}\right)$such that

$$
|G(x, s)| \geq \xi(x)|s|^{\nu}, \quad \forall x \in \Omega,
$$

where $G(x, s)=\int_{0}^{s} g(x, \tau) d \tau$, then one has the following results:

(i) if $k=1$ and $\mu<1$ with

$$
\begin{aligned}
\mu=\inf & \left\{\int_{\Omega}\left(u^{\prime \prime}(x)\right)^{2}-w u^{\prime}(x)^{2}\right. \\
& +a(x) u(x)^{2} d x \mid u \in H_{0}^{2}(\Omega), \\
& \left.\int_{\Omega} q(x) u(x)^{2} d x=1\right\},
\end{aligned}
$$

then there exists $\Lambda>0$ such that, for every $\lambda \in(0, \Lambda)$, problem (1) has at least two homoclinic solutions,

(ii) if $k>1$, then there exists $\Lambda>0$ such that, for every $\lambda \in(0, \Lambda)$, problem (1) has at least two homoclinic solutions.
The paper is organized as follows. In Section 2, we present some preliminaries. In Section 3, we give the proof of our main results.

\section{Preliminaries}

In order to prove our main results, we first give some properties of space $X$ on which the variational setting for problem (1) is defined.

Lemma 3 (see [5]). Assume that the $(A)$ holds. Then, there exists a constant $c_{0}>0$ such that

$$
\begin{gathered}
\int_{\mathbb{R}}\left[u^{\prime \prime}(x)^{2}-w u^{\prime}(x)^{2}+a(x) u(x)^{2}\right] d x \\
\geq c_{0}\|u\|_{H^{2}}^{2}, \quad \forall u \in H^{2}(\mathbb{R}),
\end{gathered}
$$

where $\|u\|_{H^{2}}^{2}=\left(\int_{\mathbb{R}}\left[u^{\prime \prime}(x)^{2}+u^{\prime}(x)^{2}+u(x)^{2}\right] d x\right)^{1 / 2}$ is the norm of Sobolev space $H^{2}(\mathbb{R})$.

Letting

$$
\begin{aligned}
& X=\{u \in H^{2}(\mathbb{R}) \mid \int_{\mathbb{R}}\left[u^{\prime \prime}(x)^{2}-w u^{\prime}(x)^{2}+a(x) u(x)^{2}\right] d x \\
&<+\infty\},
\end{aligned}
$$

then $X$ is a Hilbert space with the inner product

$$
\begin{gathered}
(u, v)=\int_{\mathbb{R}}\left[u^{\prime \prime}(x) v^{\prime \prime}(x)-w u^{\prime}(x) v^{\prime}(x)\right. \\
+a(x) u(x) v(x)] d x
\end{gathered}
$$

and the corresponding norm $\|u\|^{2}=(u, u)$. Note that

$$
X \subset H^{2}(\mathbb{R}) \subset L^{r}(\mathbb{R})
$$

for all $r \in[2,+\infty]$ with the embedding being continuous.

Lemma 4 (see [8]). Assume that the condition (A) holds. Then, $X$ is compactly embedded in $L^{r}(\mathbb{R})$ for all $r \in[2,+\infty]$.

Now, we begin describing the variational formulation of problem (1). Consider the functional $J: X \rightarrow \mathbb{R}$ defined by

$$
J(u)=\frac{1}{2}\|u\|^{2}-\int_{\mathbb{R}} F(x, u) d x-\lambda \int_{\mathbb{R}} G(x, u) d x .
$$

Since $f, g$ are continuous, by Lemma $4, J \in C^{1}(X, \mathbb{R})$ and its derivative is given by

$$
\begin{aligned}
& J^{\prime}(u) v \\
& =\int_{\mathbb{R}}\left[u^{\prime \prime}(x) v^{\prime \prime}(x)-w u^{\prime}(x) v^{\prime}(x)+a(x) u(x) v(x)\right] d x \\
& \quad-\int_{\mathbb{R}} f(x, u(x)) v(x) d x-\lambda \int_{\mathbb{R}} g(x, u(x)) v(x) d x
\end{aligned}
$$


for all $u, v \in X$. In addition, any critical point of $J$ on $X$ is a classical solution of problem (1).

Next, we give the variant version of the mountain pass theorem which is important for the proof of our main results.

Theorem 5 (see [11]). Let $E$ be a real Banach space with its dual space $E^{*}$, and suppose that $I \in C^{1}(E, \mathbb{R})$ satisfies

$$
\max \{I(0), I(e)\} \leq \mu<\eta \leq \inf _{\|u\|=\rho} I(u)
$$

for some $\mu<\eta, \rho>0$ and $e \in E$ with $\|e\|>\rho$. Let $\widehat{c} \geq \eta$ be characterized by

$$
\widehat{c}=\inf _{\gamma \in \Gamma} \max _{0 \leq \tau \leq 1} I(\gamma(\tau)),
$$

where $\Gamma=\{\gamma \in C([0,1], E): \gamma(0)=0, \gamma(1)=e\}$ is the set of continuous paths joining 0 and $e$; then, there exists a sequence $\left\{u_{n}\right\} \subset E$ such that

$$
\begin{array}{r}
I\left(u_{n}\right) \longrightarrow \widehat{c} \geq \eta, \quad\left(1+\left\|u_{n}\right\|\right)\left\|I^{\prime}\left(u_{n}\right)\right\|_{E^{*}} \\
\text { as } n \longrightarrow 0, \\
\longrightarrow \infty .
\end{array}
$$

\section{Proof of the Main Results}

To prove our main results, we first give the following lemma.

Lemma 6. For any real number $k \geq 1$, assume that conditions $(A),\left(F_{1}\right)-\left(F_{2}\right)$, and $(G)$ hold. Then, there exists $\Lambda>0$ such that for every $\lambda \in(0, \Lambda)$ there exist two positive constants $\rho, \eta$ such that $\left.J(u)\right|_{\|u\|=\rho} \geq \eta>0$.

Proof. For any $\epsilon>0$, it follows from conditions $\left(\mathrm{F}_{1}\right)-\left(\mathrm{F}_{2}\right)$ that there exist $C_{\epsilon}>0$ and $r>\max \{2, k\}$ such that

$$
F(x, s) \leq \frac{|b|_{\infty}+\epsilon}{2} s^{2}+\frac{C_{\epsilon}}{r}|s|^{r}, \quad \forall s \in \mathbb{R} .
$$

By (9) and (21), the Sobolev inequality, and the Hölder inequality, one has, for all $u \in X$,

$$
\begin{aligned}
J(u)= & \frac{1}{2}\|u\|^{2}-\int_{\mathbb{R}} F(x, u) d x-\lambda \int_{\mathbb{R}} G(x, u) d x \\
\geq & \frac{1}{2}\|u\|^{2}-\int_{\mathbb{R}} \frac{|b|_{\infty}+\epsilon}{2} u(x)^{2} d x \\
& -\int_{\mathbb{R}} \frac{C_{\epsilon}}{r} u(x)^{r} d x-\lambda \int_{\mathbb{R}} h(x)|u(x)|^{p} d x \\
\geq & \frac{1}{2}\|u\|^{2}-\frac{|b|_{\infty}+\epsilon}{2 c_{0} S_{2}^{2}}\|u\|^{2}-\frac{C_{\epsilon}}{r c_{0}^{r / 2} S_{r}^{r}}\|u\|^{r} \\
& -\lambda c_{0}^{-p / 2} S_{2}^{-p}\|h\|_{2 /(2-p)}\|u\|^{p} \\
= & \|u\|^{p}\left[\frac{1}{2}\left(1-\frac{|b|_{\infty}+\epsilon}{2 c_{0} S_{2}^{2}}\right)\|u\|^{2-p}-\frac{C_{\epsilon}}{r c_{0}^{r / 2} S_{r}^{r}}\|u\|^{r-p}\right. \\
& \left.-\lambda c_{0}^{-p / 2} S_{2}^{-p}\|h\|_{2 /(2-p)}\right] .
\end{aligned}
$$

Take $\epsilon=\left(c_{0} S_{2}^{2} / 2\right)-|b|_{\infty}$ and define

$$
l(t)=\frac{1}{4} t^{2-p}-C_{\epsilon} r^{-1} c_{0}^{-r / 2} S_{r}^{-r} t^{r-p}, \quad \forall t \geq 0 .
$$

It is easy to prove that there exists $\rho>0$ such that

$$
\max _{t \geq 0} l(t)=l(\rho)=\frac{r-2}{4(r-p)}\left[\frac{(2-p) r c_{0}^{r / 2} S_{r}^{r}}{4(r-p) C_{\epsilon}}\right]^{(2-p) /(r-2)} .
$$

Then, it follows from (22) that there exists $\Lambda>0$ such that for every $\lambda \in(0, \Lambda)$ there exist two positive constants $\rho, \eta$ such that $\left.J(u)\right|_{\|u\|=\rho} \geq \eta>0$.

Consider the minimum problem

$$
\begin{gathered}
\mu=\inf \left\{\int _ { \Omega } \left[u^{\prime \prime}(x)^{2}-w u^{\prime}(x)^{2}\right.\right. \\
\left.\quad+a(x) u(x)^{2}\right] d x \mid u \in H_{0}^{2}(\Omega), \\
\left.\int_{\Omega} q(x) u(x)^{2} d x=1\right\} .
\end{gathered}
$$

Then, we have the following results.

Lemma 7. There exist a constant $c_{1}>0$ and $\phi_{1} \in H_{0}^{2,2}(\Omega)$ with $\int_{\Omega} q(x) \phi_{1}(x)^{2} d x=1$ such that $\mu \geq c_{1}$ and

$$
\mu=\int_{\Omega}\left[\left(\phi_{1}^{\prime \prime}(x)\right)^{2}-w \phi_{1}^{\prime}(x)^{2}+a(x) \phi_{1}(x)^{2}\right] d x
$$

that is, the minimum (25) is achieved.

Proof. For any $u \in H_{0}^{2,2}(\Omega)$ with $\int_{\Omega} q(x) u(x)^{2} d x=1$, by Lemma 3 and the Sobolev embedded theorem, we have

$$
\begin{gathered}
\int_{\Omega}\left[u^{\prime \prime}(x)^{2}-w u^{\prime}(x)^{2}+a(x) u(x)^{2}\right] d x \\
\geq c_{0}\|u\|_{H_{0}^{2}}^{2} \geq c_{0} S_{2}^{2}\|u\|_{2}^{2} \geq \frac{c_{0} S_{2}^{2}}{|q|_{\infty}}>0 .
\end{gathered}
$$

Therefore, there exists a constant $c_{1}>0$ such that $\mu \geq c_{1}$. Let $\left\{u_{n}\right\} \subset H_{0}^{2}(\Omega)$ be a minimizing sequence of (25). Clearly, $\int_{\Omega} q(x) u_{n}(x)^{2} d x=1$ and $\left\{u_{n}\right\}$ is bounded. Then, there exist a subsequence $\left\{u_{n}\right\}$ and $\phi_{1} \in H_{0}^{2}(\Omega)$ such that $u_{n} \rightarrow \phi_{1}$ weakly in $H_{0}^{2}(\Omega)$ and $u_{n} \rightarrow \phi_{1}$ strongly in $L^{2}(\Omega)$. So it is easy to verify that $\int_{\Omega} q(x) u_{n}(x)^{2} d x \rightarrow \int_{\Omega} q(x) \phi_{1}(x)^{2} d x$ as $n \rightarrow \infty$ and $\int_{\Omega} q(x) \phi_{1}(x)^{2} d x=1$. Therefore,

$$
\begin{aligned}
\mu & \leq \int_{\Omega}\left(\phi_{1}^{\prime \prime}(x)\right)^{2}-w \phi_{1}^{\prime}(x)^{2}+a(x) \phi_{1}(x)^{2} d x \\
& \leq \liminf _{n \rightarrow \infty} \int_{\Omega}\left[u_{n}^{\prime \prime}(x)^{2}-w u_{n}^{\prime}(x)^{2}+a(x) u_{n}(x)^{2}\right] d x \\
& \leq \mu .
\end{aligned}
$$


This implies that

$$
\mu=\int_{\Omega}\left(\phi_{1}^{\prime \prime}(x)\right)^{2}-w \phi_{1}^{\prime}(x)^{2}+a(x) \phi_{1}(x)^{2} d x .
$$

Lemma 8. For any real number $k \geq 1$, assume that conditions $(A),\left(F_{1}\right)-\left(F_{2}\right)$, and $\left(G_{1}\right)-\left(G_{2}\right)$ hold. Let $\rho, \Lambda>0$ be as in Lemma 6. Then, one has the following results.

(i) If $k=1$ and $\mu<1$, then there exists $e \in X$ with $\|e\|>\rho$ such that $J(e)<0$ for all $\lambda \in(0, \Lambda)$.

(ii) If $k>1$, then there exists $e \in X$ with $\|e\|>\rho$ such that $J(e)<0$ for all $\lambda \in(0, \Lambda)$.

Proof. (i) In case $k=1$. Since $\mu<1$, we can choose a nonnegative function $\varphi \in H_{0}^{2}(\Omega)$ with $\int_{\Omega} q(x) \varphi(x)^{2} d x=1$ such that

$$
\int_{\Omega}\left[\varphi^{\prime \prime}(x)^{2}-w \varphi^{\prime}(x)^{2}+a(x) \varphi(x)^{2}\right] d x<1 .
$$

Therefore, by condition $\left(\mathrm{F}_{2}\right)$ and the Fatou lemma, we have

$$
\begin{aligned}
\lim _{t \rightarrow+\infty} & \frac{J(t \varphi)}{t^{2}} \\
\leq & \frac{1}{2}\|\varphi\|^{2}-\lim _{t \rightarrow+\infty} \int_{\Omega} \frac{F(x, t \varphi)}{t^{2} \varphi^{2}} \varphi^{2} d x \\
& -\lim _{t \rightarrow+\infty} \frac{\lambda}{t^{2-\nu}} \int_{\Omega} \xi(x)|\varphi|^{\nu} d x \\
\leq & \frac{1}{2}\|\varphi\|^{2}-\frac{1}{2} \int_{\Omega} q(x) \varphi^{2} d x \\
= & \frac{1}{2}\left(\|\varphi\|^{2}-1\right)<0 .
\end{aligned}
$$

So, $J(t \varphi) \rightarrow-\infty$ as $t \rightarrow+\infty$; then, there exists $e \in X$ with $\|e\|>\rho$ such that $J(e)<0$ for all $\lambda \in(0, \Lambda)$.

(ii) In case $k>1 . q \in L^{\infty}\left(\mathbb{R}, \mathbb{R}^{+}\right)$with $q^{+} \neq 0$ in $\Omega$, and we can choose a function $\omega \in H_{0}^{2}(\Omega)$ such that

$$
\int_{\Omega} q(x)|\omega|^{k+1} d x>0 .
$$

Therefore, by condition $\left(\mathrm{F}_{2}\right)$ and the Fatou lemma, we have

$$
\begin{aligned}
\lim _{t \rightarrow+\infty} \frac{J(t \omega)}{t^{k+1}} \leq & \frac{\|\omega\|^{2}}{2 t^{k-1}}-\lim _{t \rightarrow+\infty} \int_{\Omega} \frac{F(x, t \omega)}{t^{k+1} \omega^{k+1}} \varphi^{k+1} d x \\
& -\lim _{t \rightarrow+\infty} \frac{\lambda}{t^{k+1-\gamma}} \int_{\Omega} \xi(x)|\omega|^{v} d x \\
\leq & -\frac{1}{k+1} \int_{\Omega} q(x) \omega^{k+1} d x \\
& <0 .
\end{aligned}
$$

So, $J(t \omega) \rightarrow-\infty$ as $t \rightarrow+\infty$; then, there exists $e \in X$ with $\|e\|>\rho$ such that $J(e)<0$ for all $\lambda \in(0, \Lambda)$.

Next, we define

$$
\beta=\inf _{\gamma \in \Gamma} \max _{0 \leq \tau \leq 1} J(\gamma(\tau)),
$$

where $\Gamma=\{\gamma \in C([0,1], E): \gamma(0)=0, \gamma(1)=e\}$. Then, by Theorem 5 and Lemmas 6 and 8 , there exists a sequence $\left\{u_{n}\right\} \subset X$ such that

$$
\begin{array}{r}
J\left(u_{n}\right) \longrightarrow \beta, \quad\left(1+\left\|u_{n}\right\|\right)\left\|J^{\prime}\left(u_{n}\right)\right\|_{E^{*}} \\
\text { as } n \longrightarrow 0, \\
\longrightarrow \infty .
\end{array}
$$

Then, we have the following results.

Lemma 9. For any real number $k \geq 1$, assume that conditions $(A),\left(F_{1}\right)-\left(F_{3}\right)$, and $\left(G_{1}\right)-\left(G_{2}\right)$ hold. Let $\Lambda>0$ be as in Lemma 6. Then, $\left\{u_{n}\right\}$ defined by (35) is bounded in $X$ for all $\lambda \in(0, \Lambda)$.

Proof. For $n$ large enough, by the Hölder inequality and Lemma 3, one has

$$
\begin{aligned}
\beta+1 \geq & J\left(u_{n}\right)-\frac{1}{\theta}\left\langle J^{\prime}\left(u_{n}\right), u_{n}\right\rangle \\
= & \left(\frac{1}{2}-\frac{1}{\theta}\right)\left\|u_{n}\right\|^{2}-\int_{\mathbb{R}}\left[F\left(x, u_{n}\right)-\frac{1}{\theta} f\left(x, u_{n}\right) u_{n}\right] d x \\
& -\lambda \int_{\mathbb{R}}\left[G\left(x, u_{n}\right)-\frac{1}{\theta} g\left(x, u_{n}\right) u_{n}\right] d x \\
\geq & \frac{\theta-2}{2 \theta}\left\|u_{n}\right\|^{2}-d_{0} \int_{\mathbb{R}} u_{n}^{2} d x \\
& -\lambda\left(1+\frac{p}{\theta}\right) \int_{\mathbb{R}} h(x) u_{n}^{p} d x \\
\geq & \frac{\theta-2}{2 \theta}\left\|u_{n}\right\|^{2}-\frac{d_{0}}{c_{0} S_{2}^{2}}\left\|u_{n}\right\|^{2} \\
& -\lambda\left(1+\frac{p}{\theta}\right) S_{2}^{-p}|h|_{2 /(2-p)}\left\|u_{n}\right\|^{p} \\
\geq & \left(\frac{\theta-2}{2 \theta}-\frac{d_{0}}{c_{0} S_{2}^{2}}\right)\left\|u_{n}\right\|^{2} \\
& -\Lambda\left(1+\frac{p}{\theta}\right) S_{2}^{-p}|h|_{2 /(2-p)}\left\|u_{n}\right\|^{p}
\end{aligned}
$$

which implies that $\left\{u_{n}\right\}$ is bounded in $X$, since $1 \leq p<2$.

For $\rho$ given by Lemma 6 , denote $B_{\rho}=\{u \in X:\|u\|<\rho\}$. Then, by Ekeland's variational principle and Lemma 4, we have the following lemma, which shows that $J$ has a local minimum if $\lambda$ is small.

Lemma 10. For any real number $k \geq 1$, assume that conditions $(A),\left(F_{1}\right)-\left(F_{3}\right)$, and $\left(G_{1}\right)-\left(G_{2}\right)$ hold. Let $\Lambda>0$ be as in Lemma 6. Then, for every $\lambda \in(0, \Lambda)$, there exists $u_{0} \in X$ such that

$$
J\left(u_{0}\right)=\inf \left\{J(u): u \in \bar{B}_{\rho}\right\}<0
$$

and $u_{0}$ is a homoclinic solution of problem (1). 
Proof. Since $\xi \in L^{\infty}\left(\mathbb{R}, \mathbb{R}^{+}\right)$with $\xi^{+} \neq 0$ in $\Omega$, we can choose a function $\psi \in H_{0}^{2}(\Omega)$ such that

$$
\int_{\Omega} \xi(x)|\psi|^{\nu} d x>0 .
$$

Hence, we have

$$
\begin{aligned}
J(t \psi)= & \frac{t^{2}}{2}\|\psi\|^{2}-\int_{\Omega} F(x, t \psi) d x \\
& -\lambda t^{\nu} \int_{\Omega} \xi(x)|\psi|^{\nu} d x \\
\leq & \frac{t^{2}}{2}\|\psi\|^{2}-\lambda t^{\nu} \int_{\Omega} \xi(x)|\psi|^{\nu} d x<0
\end{aligned}
$$

for $t>0$ small enough. This implies $\theta_{0}:=\inf \{J(u): u \epsilon$ $\left.\bar{B}_{\rho}\right\}<0$. By the Ekeland variational principle, there exists a minimizing sequence $\left\{u_{n}\right\} \subset \bar{B}_{\rho}$ such that $J\left(u_{n}\right) \rightarrow \theta_{0}$ and $J^{\prime}\left(u_{n}\right) \rightarrow 0$ as $n \rightarrow \infty$. Hence, Lemma 4 implies that there exists $u_{0} \in X$ such that $J^{\prime}\left(u_{0}\right)=0$ and $J\left(u_{0}\right)=c_{1}<0$.

Proof of Theorem 2. From Lemmas 4 and 8 , there is $\bar{u} \in X$ such that, up to a subsequence, $u_{n} \rightarrow \bar{u}$ weakly in $X$ and $u_{n} \rightarrow \bar{u}$ strongly in $L^{s}(\mathbb{R})$ for $s \in[2, \infty]$. By using a standard procedure, we can prove that $u_{n} \rightarrow \bar{u}$ strongly in $X$. Moreover, $J(\bar{u})=\beta>0$ and $\bar{u}$ is another homoclinic solution of problem (1). Therefore, combining with Lemma 10, we prove that problem (1) has at least two homoclinic solutions $u_{0}, \bar{u} \in X$ satisfying $J\left(u_{0}\right)<0$ and $J(\bar{u})>0$.

\section{Conflict of Interests}

The author declares that there is no conflict of interests regarding the publication of this paper.

\section{Acknowledgment}

The paper was supported by NFSC (11271372).

\section{References}

[1] Y. Chen and P. J. McKenna, “Traveling waves in a nonlinearly suspended beam: theoretical results and numerical observations," Journal of Differential Equations, vol. 136, no. 2, pp. 325355, 1997.

[2] B. Buffoni, "Periodic and homoclinic orbits for LorentzLagrangian systems via variational methods," Nonlinear Analysis: Theory, Methods \& Applications, vol. 26, no. 3, pp. 443-462, 1996.

[3] D. Smets and J. C. van den Berg, "Homoclinic solutions for Swift-Hohenberg and suspension bridge type equations," Journal of Differential Equations, vol. 184, no. 1, pp. 78-96, 2002.

[4] S. Santra and J. Wei, "Homoclinic solutions for fourth order traveling wave equations," SIAM Journal on Mathematical Analysis, vol. 41, no. 5, pp. 2038-2056, 2009.

[5] S. Tersian and J. Chaparova, "Periodic and homoclinic solutions of extended Fisher-Kolmogorov equations," Journal of Mathematical Analysis and Applications, vol. 260, no. 2, pp. 490-506, 2001.
[6] Y. L. Yeun, "Heteroclinic solutions for the extended FisherKolmogorov equation," Journal of Mathematical Analysis and Applications, vol. 407, no. 1, pp. 119-129, 2013.

[7] C. Li, "Remarks on homoclinic solutions for semilinear fourthorder ordinary differential equations without periodicity," Applied Mathematics, vol. 24, no. 1, pp. 49-55, 2009.

[8] J. Sun and T. Wu, "Two homoclinic solutions for a nonperiodic fourth order differential equation with a perturbation," Journal of Mathematical Analysis and Applications, vol. 413, no. 2, pp. 622-632, 2014.

[9] P. Coullet, C. Elphick, and D. Repaux, "Nature of spatial chaos," Physical Review Letters, vol. 58, no. 5, pp. 431-434, 1987.

[10] J. Lega, J. V. Moloney, and A. C. Newell, "Swift-Hohenberg equation for lasers," Physical Review Letters, vol. 73, no. 22, pp. 2978-2981, 1994.

[11] I. Ekeland, Convexity Methods In Hamiltonian Mechanics, vol. 19, Springer, Berlin, Germany, 1990. 


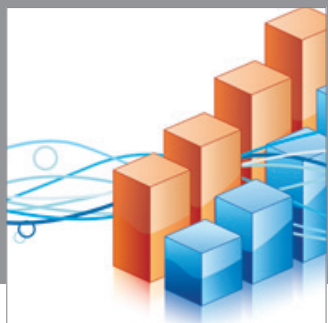

Advances in

Operations Research

mansans

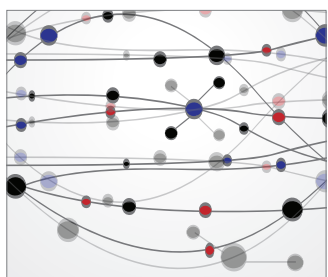

The Scientific World Journal
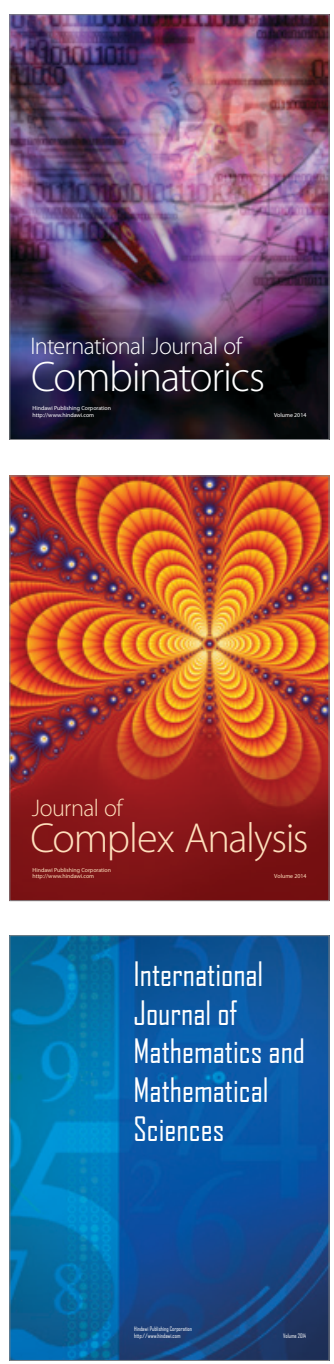
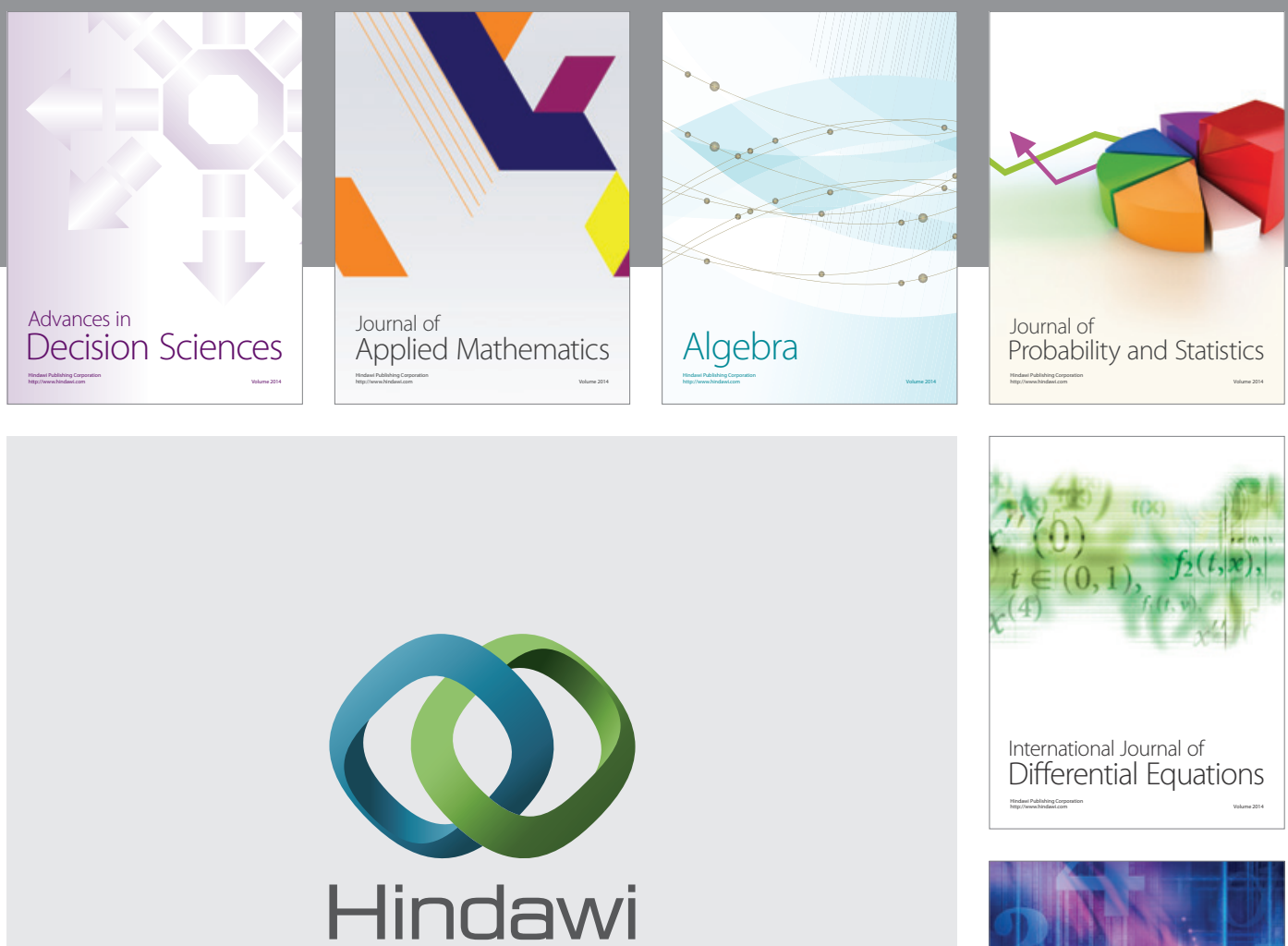

Submit your manuscripts at http://www.hindawi.com
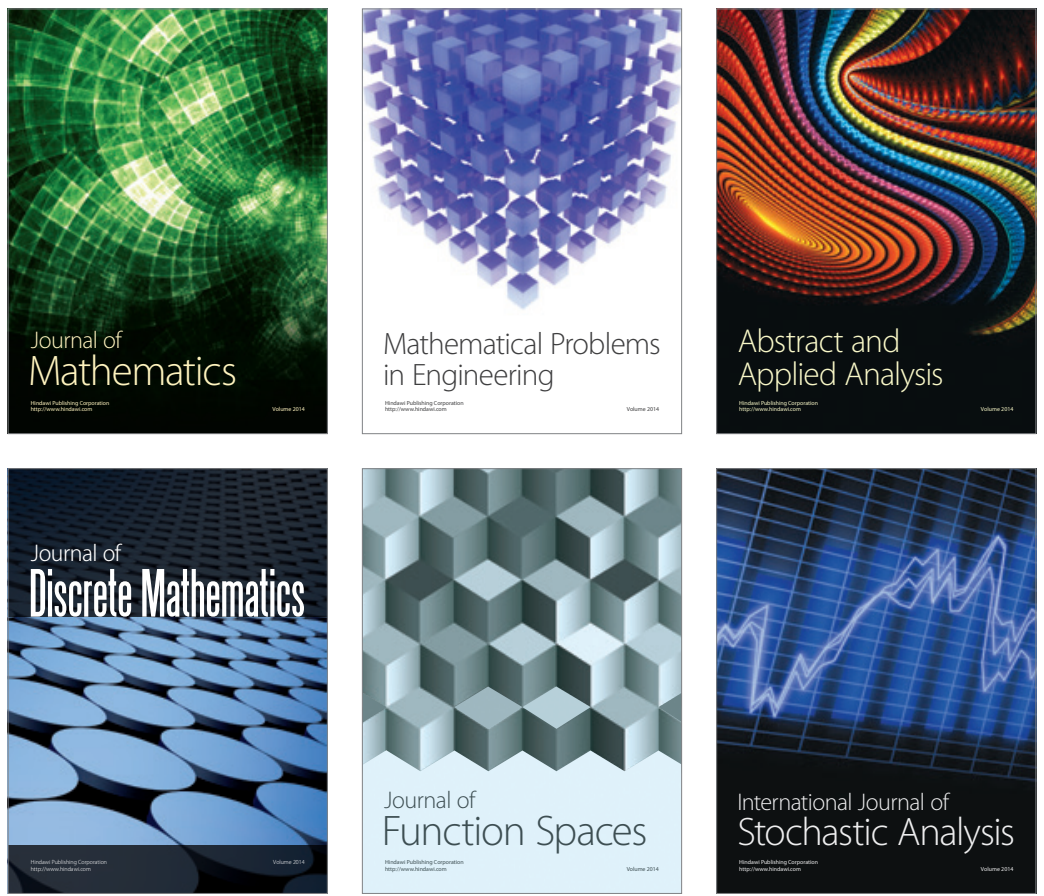

Journal of

Function Spaces

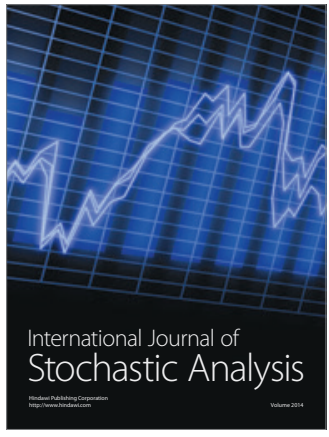

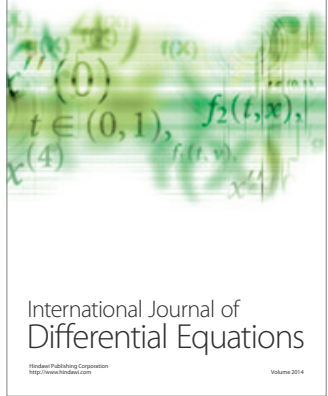
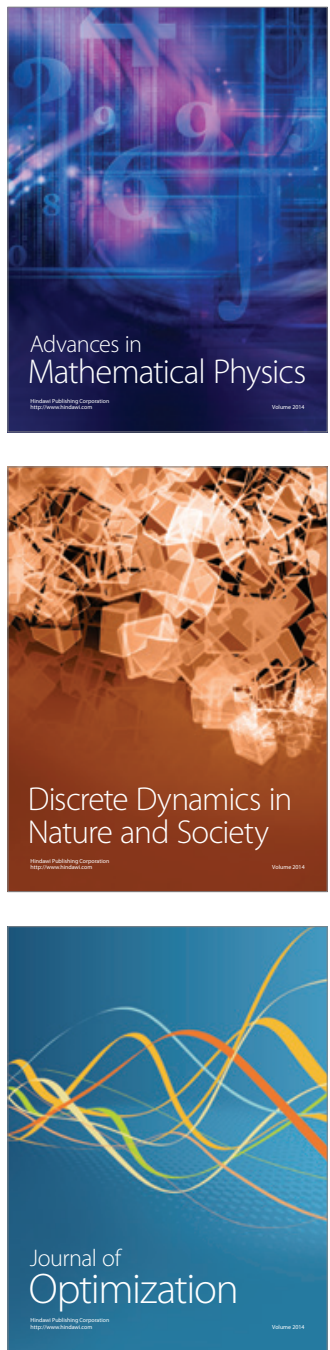